\title{
MicroRNA-186-5p mediates osteoblastic differentiation and cell viability by targeting CXCL13 in non-traumatic osteonecrosis
}

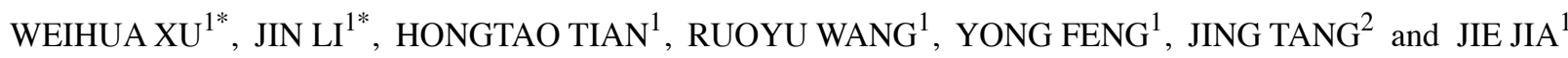 \\ ${ }^{1}$ Department of Orthopedics; ${ }^{2}$ Cancer Centre, Union Hospital, Tongji Medical College, \\ Huazhong University of Science and Technology, Wuhan, Hubei 430022, P.R. China
}

Received February 21, 2019; Accepted July 19, 2019

DOI: $10.3892 / \mathrm{mmr} .2019 .10710$

\begin{abstract}
MicroRNAs (miRs) serve varying and important roles in the pathogenesis of non-traumatic osteonecrosis $(\mathrm{ON})$. However, the role miR-186-5p serves in the pathogenesis of osteonecrosis remains unknown and the clinical outcome of ON is still uncertain. The aim of the present study was to determine the expression characteristics, biological function and molecular mechanisms of miR-186-5p, which is associated with cancer development and progression, in osteoblastic differentiation and cell viability. The results of the present study showed that the expression levels of miR-186-5p were significantly higher in clinical non-traumatic ON compared with osteoarthritis samples $(\mathrm{P}=0.0001)$. An inverse association was observed between miR-186-5p and CXCL13 expression levels. Furthermore, miR-186-5p inhibited phosphatidylinositol 3 kinase (PI3K)/protein kinase B (AKT) signaling, downregulated osteoblast-specific markers and reduced the viability and differentiation of human mesenchymal stem cells from bone marrow (HMSC-bm) through targeting CXCL13. Increasing expression of CXCL13 in HMSC-bm cells partially restored miR-186-5p-mediated inhibition. In conclusion, abrogation of PI3K/AKT signaling triggered by miR-186-5p/CXCL13 may contribute to ON pathogenesis. These results highlight the possible clinical value of miR-186-5p in treatment for non-traumatic ON.
\end{abstract}

Correspondence to: Dr Jie Jia, Department of Orthopedics, Union Hospital, Tongji Medical College, Huazhong University of Science and Technology, 1277 Jiefang Avenue, Wuhan, Hubei 430022, P.R. China E-mail: jjwhunion@163.com

Dr Jing Tang, Cancer Centre, Union Hospital, Tongji Medical College, Huazhong University of Science and Technology, 1277 Jiefang Avenue, Wuhan, Hubei 430022, P.R. China

E-mail: michelleyiyi@hotmail.com

${ }^{*}$ Contributed equally

Key words: microRNA, miR-186-5p, CXCL13, phosphatidylinositol 3 kinase/protein kinase B, non-traumatic osteonecrosis, osteogenic differentiation

\section{Introduction}

Non-traumatic osteonecrosis $(\mathrm{ON})$ arises from increased intraosseous pressure caused by fat cell hypertrophy and abnormal adipogenesis in the bone marrow, which subsequently results in ischemia which leads to osteocyte and bone marrow cell (BMC) death (1). The incidence of $\mathrm{ON}$ has been increasing, especially in men between 30 and 50 years of age (2-4). The underlying pathogenesis of $\mathrm{ON}$ is multifactorial, including adipogenesis or fat hypertrophy, imbalance of osteoblasts, angiogenesis inhibition and apoptosis of endothelial cells (5-7). ON therapy primarily includes conventional core decompression and joint replacement surgery. However, this treatment only achieves a moderate decrease in intra-medullary pressure, with few clinical benefits and a high expense (8).

Based on the theory that mesenchymal stem cells (MSCs) are pluripotent cells which can differentiate into a range of cell types, transplantation of MSCs is used as a novel treatment strategy. For example, implantation of autologous BMCs has been successfully applied in treating early stages of ON $(9,10)$. However, several studies indicate that decreased osteogenic differentiation and replication capacity of MSCs in the bone marrow also have crucial roles in non-traumatic ON (11), and abnormal MSCs are observed after transplantation of MSCs $(12,13)$, thus limiting its application.

MicroRNAs (miRNAs/miRs) are small, endogenous, noncoding RNAs that potentially regulate a large number of genes (14-16). miRNAs are thought to modulate $30 \%$ of the human genome through base-pairing with 3'untranslated regions (UTRs) of their target mRNAs and ultimately cleaving mRNA or repressing translation mRNAs (14-17). Although miRNAs serve as regulators in a number of biological processes, including morphogenesis, differentiation, proliferation and tumorigenesis (18), there are relatively fewer reports examining the role of miRNA in the pathogenesis of ON $(19,20)$.

miR-186 has been reported to serve as an inhibitor of tumor cell growth and migration in various types of cancer such as lung cancer $(21,22)$ and colorectal cancer $(23)$. In the present study, the role of miR-186-5p in osteoblastic differentiation and cell growth of ON patients was determined. miR-186-5p expression was relatively higher in MSCs of patients with non-traumatic $\mathrm{ON}$ and inhibited the viability and differentiation of osteoblastic cells in human mesenchymal stem cells 
(HMSCs). CXCL13 was a functional target of miR-186-5p in HMSCs. In addition, miR-186-5p modulated protein kinase B (AKT) signaling in HMSCs. These findings suggest that miR-186-5p may be a potential molecular target for treating patients with non-traumatic $\mathrm{ON}$.

\section{Materials and methods}

Clinical samples. The present study was approved by the Ethics Committee of Wuhan Union Hospital, bone marrow samples were obtained with informed consent from $15 \mathrm{ON}$ patients and 15 osteoarthritis (OA) patients receiving surgery between October 2010 and July 2013 at Wuhan Union Hospital, Huazhong University of Science and Technology. Patient characteristics are shown in Table I.

Bone marrow samples of 5-10 $\mathrm{ml}$ from the proximal end of the femur of each patient were taken by inserting a tapered awl into the femoral canal during total hip arthroplasty. OA was diagnosed using radiographic images, and the diagnostic criteria were satisfied for all patients. Non-traumatic ON was confirmed based on radiographic and magnetic resonance imaging. Of the 15 patients with non-traumatic ON, nine were treated with corticosteroids, five suffered from alcoholism and one was diagnosed as idiopathic ON. The clinical characteristics of patients are shown in Table I. To obtain mesenchymal stem cells (MSCs), mononuclear cells from the bone marrow were obtained using Ficoll-Hypaque gradient centrifugation (Fresenius Kabi I Norge AG), suspended in low-glucose Dulbecco's modified Eagle's medium (DMEM; HyClone; GE Healthcare Life Sciences) containing 10\% fetal bovine serum (FBS; Gibco: Thermo Fisher Scientific, Inc.) and seeded at a density of $1 \times 10^{6}$ cells per $\mathrm{cm}^{2}$.

Cell culture. 293T cells and HMSC-bm cells were obtained from the Type Culture Collection of the Chinese Academy of Sciences and cultured in MSC growth medium consisting of low-glucose DMEM supplemented with 10\% FBS and maintained in a cell incubator at $37^{\circ} \mathrm{C}$ with $5 \% \mathrm{CO}_{2}$. Cells were cultured in flasks and upon reaching $90 \%$ confluency, cells were passaged at 1:2 dilution. Cells between the 4 and 7 th passages were used for the following experiments. Osteoblastic differentiation was accomplished using medium containing 100 ng/ml BMP-2 (R\&D Systems China, Co., Ltd.) and $10 \%$ FBS. For AKT activation, cells were incubated with $10 \mathrm{mM} \mathrm{LiCl}$ (7447-41-8, ApexBio, Houston, TX) for $48 \mathrm{~h}$ after transfection.

RNA-seq and data analysis. RNA sequencing was performed at Guangzhou RiboBio Co., Ltd., using an Illumina HiSeq 2500. RNA-seq data was aligned to the Ensembl transcript annotations (GenBank Assembly ID GCA_000001405.25) using bowtie and RSEM as previously described (24). Cufflinks (version 2.2.1) (25) was used to estimate gene expression abundance (number of fragments per $\mathrm{kb}$ of each gene per million mapped reads). Differentially expressed genes were detected using DESeq (version 1.16.0) (26) and a false discovery rate $<0.01$ and a fold-change $>1.25$ were used as thresholds to define significantly differentially expressed genes. Other bioinformatics analyses were performed using glbase (27) and additional; details are provided in Fig. S1 and Table SIII.
Kyoto Encyclopedia of Genes and Genomes (KEGG) pathway analysis was employed to determine the significant pathways (http://www.genome.jp/kegg/).

MTT assays. For the cell viability assay, cells were seeded into a 96-well plate $\left(1 \times 10^{3}\right.$ cells/well) and used to perform MTT assays (Sigma-Aldrich; Merck KGaA). MTT diluted in PBS (5 $\mathrm{mg} / \mathrm{ml})$ was added to each well $(20 \mu \mathrm{l}$ per well) and incubated for $4 \mathrm{~h}$. Formazan was then dissolved in DMSO (200 $\mu \mathrm{l}$ per well) and the absorbance value at $490 \mathrm{~nm}$ (optical density, $490 \mathrm{~nm}$ ) was determined.

Reverse transcription-quantitative PCR (RT-qPCR). The total RNA of samples or cells was extracted using a miRNeasy mini kit or RNeasy kit (Qiagen China Co., Ltd.). RNA was reverse transcribed using Transcriptor (Roche Diagnostics) with an oligo-dT primer or the Bulge-loop ${ }^{\mathrm{TM}}$ miRNA qRT-PCR Primer Sets specific for miR-miR-186-5p or U6 (Guangzho Ribobio Co., Ltd.) according to the manufacturer's protocol. Briefly, the reactions were incubated for $60 \mathrm{~min}$ at $42^{\circ} \mathrm{C}$ and $10 \mathrm{~min}$ at $70^{\circ} \mathrm{C}$ and were then stored at $-20^{\circ} \mathrm{C}$. RT-qPCR analysis was performed using a LightCycler 96 System (Roche Diagnostics) with SYBR Green PCR Master Mix (Takara Bio, Inc.). In brief, the reactions were incubated at $95^{\circ} \mathrm{C}$ for $20 \mathrm{sec}$, followed by 40 cycles of $95^{\circ} \mathrm{C}$ for $10 \mathrm{sec}$ and $60^{\circ} \mathrm{C}$ for $35 \mathrm{sec}$. A dissociation stage was performed at the end of the amplification procedure to determine potential non-specific amplifications. U6 and GAPDH were used as the internal control. Relative gene expression in functional assay was analyzed using the $2^{-\Delta \Delta \mathrm{Cq}}$ method (28) and present with fold-changes. Relative gene expression in results of clinical samples was present with $-\Delta \mathrm{Cq}[\Delta \mathrm{Cq}=\mathrm{Cq}$ (Gene)-Cq (U6)]. Primer used for RT-qPCR were listed in Table SI.

Transfection. To determine the effects of miR-186-5p on cellular function, a miR-186-5p mimic, miRNA mimic control, anti-miR-186-5p, nonspecific anti-miRNA control and small interfering (si)RNAs targeting human CXCL13 (si-CXCL13) were all synthesized by Guangzhou RiboBio Co., Ltd. For transfection, INTERFER in ${ }^{\circledR}$ (Polyplus-transfection SA) and $20 \mathrm{nM}$ of the RNAs mentioned above were prepared according to the manufacturer's protocol and mixed with cells in 24-well cell culture plates when the cells were at $50 \%$ confluence. The medium was replaced with fresh medium $12 \mathrm{~h}$ after transfection.

MicroRNA target prediction and luciferase reporter assays. TargetScan version 7.2 (http://www.targetscan.org/vert_72/) was used to predict the targets of miR-186-5p. The complete computational protocol is available at the above website. The miR-186-5p target region containing the CXCL13 3'UTR (5'-GGACUCUGGUAUCUAAUUCUUUA-3') was inserted into the pmirGLO luciferase reporter vector (Promega Corporation). As a control, a plasmid containing mutant sites (sequence 5'-GGACUCUGGUAUCUAUAAGAAAA-3') of the miR-186-5p target region in the CXCL13 3'UTR was also inserted into the pmirGLO luciferase reporter vector. Co-transfection of $200 \mathrm{ng}$ wild type (WT) or mutant (mut) CXCL13 3'UTR with $100 \mathrm{nM}$ of miR-186-5p mimic or anti-miR-186-5p and their respective negative controls into 
Table I. Clinical characteristics of patients.

\begin{tabular}{lccc}
\hline Group & Number of patients & Age, years & Sex, F/M \\
\hline Osteoarthritis & 15 & $52 \pm 6$ & $8 / 7$ \\
Osteonecrosis & & & \\
Alcohol & 5 & $53 \pm 10$ & $1 / 4$ \\
Steroid & 9 & $46 \pm 14$ & $4 / 5$ \\
Idiopathic & 1 & 52 & $1 / 0$ \\
\hline
\end{tabular}

F, female; M, male.

293T cells was performed using INTERFERin ${ }^{\circledR}$ in 48 -well plates. Relative luciferase activity was analyzed using the dual-luciferase reporter system (Promega Corporation) and normalized with Renilla luciferase activity. Each experiment was repeated four times.

Western blotting. Western blotting was performed as described previously (29). Briefly, whole-cell extracts were prepared in lysis buffer $(20 \mathrm{mM}$ Tris- $\mathrm{HCl}, \mathrm{pH} 7.4,150 \mathrm{mM}$ $\mathrm{NaCl}, 10 \%$ glycerol, $0.2 \%$ Nonidet P-40, 1 mM EDTA, $1 \mathrm{mM}$ EGTA, $1 \mathrm{mM}$ PMSF, $10 \mathrm{mM} \mathrm{NaF}, 5 \mathrm{mg} / \mathrm{ml}$ aprotinin, $20 \mathrm{mM}$ leupeptin, and $1 \mathrm{mM}$ sodium orthovanadate) and centrifuged at $13,000 \mathrm{x} \mathrm{g}$ for $20 \mathrm{~min}$ at $4^{\circ} \mathrm{C}$. Protein concentrations were determined using the bicinchoninic acid (BCA, Invitrogen; Thermo Fisher Scientific, Inc.) assay. Immunoblotting was performed using $30 \mu \mathrm{g}$ protein lysate with specific primary antibodies (CXCL13: Proteintech 10927-1-AP; AKT: Proteintech 10176-2-AP; p-AKT (Ser473): CST 4060; ERK1/2: Proteintech 16443-1-AP; p-ERK1/2: CST 4370;) which were diluted in a SignalBoost immunoreaction enhancer buffer (Millipore, 407207-1) at $4^{\circ} \mathrm{C}$ overnight. Immunocomplexes were incubated with the fluorescein-conjugated secondary antibody (Thermo Fisher Scientific, Inc., A32735 and A32730) at room temperature for $2 \mathrm{~h}$ and then detected using an Odyssey fluorescence scanner (Li-Cor).

Osteogenic differentiation and evaluation. Osteogenic differentiation was evaluated using alkaline phosphatase (ALP) staining and osteoblast-specific gene analyses. For ALP staining, adherent cells were prefixed with $4 \%$ formaldehyde for $30 \mathrm{~min}$ and Western blue stabilized substrate was added (Promega Corporation) for $30 \mathrm{~min}$ at room temperature. ALP activity was examined using p-nitrophenyl phosphate (Sigma-Aldrich; Merck KGaA) as described previously (30). For Alizarin Red S (ARS) staining, the cells were washed with PBS and fixed with $4 \%$ formaldehyde for $30 \mathrm{~min}$ and then stained for $20 \mathrm{~min}$ with $40 \mathrm{mM}$ ARS solution (Merck Millipore, TMS-008-C). Finally, they were rinsed three times with PBS to reduce nonspecific staining. The absorbance value at $570 \mathrm{~nm}$ was measured to quantify the osteogenic differentiation. Expression levels of osteoblast-specific genes, such as RUNX2 and COL1A1 were also determined using RT-qPCR. Measurements were performed in three times for each experiment.

Statistical analysis. Data are presented as the mean \pm standard deviation of at least three independent repeats. Differences between groups were compared using analysis of variance followed by a Tukey's post-hoc test when applicable, while continuous variables were compared using Student t-test. To assess the correlation between miR-186-5p and CXCL13 expression levels, Pearson's rank correlation coefficient was used. Statistical analyses were performed with using IBM SPSS statistics version 19 (IBM Corp). $\mathrm{P}<0.05$ was considered to indicate a statistically significant difference.

\section{Results}

miR-186-5p expression is significantly increased in ON of formal head (ONFH) and modulates MSC growth and differentiation. To identify novel functional miRNAs in ONFH progression, miR-186-5p expression levels were determined in HMSCs from patients with OA and non-traumatic ON. RT-qPCR analysis showed that miR-186-5p levels were significantly increased in HMSCs from the ON group Compared with the patients with $\mathrm{OA}(\mathrm{P}<0.0001$; Fig. 1A). To determine the underlying roles of miR-186-5p during osteoblastic differentiation of HMSCs, the expression of miR-186-5p was analyzed in HMSC-bm. As shown in Fig. 1B, compared with MSCs from the negative control group, miR-186-5p expression levels increased at a slower rate when treated with BMP-2 for 6 days, suggesting that miR-186-5p may affect cell viability during MSC differentiation. These results also indicated that during growth and differentiation of HMSCs cells, the expression of miR-186-5p was elevated and cell differentiation and viability was negatively regulated by miR-186-5p. When the cells reached confluence, miR-186-5p expression levels decreased. Consistent with a previous study (21) showing that miR-186-5p negatively modulates cell proliferation, overexpression of miR-186-5p significantly suppressed HMSC-bm viability $(\mathrm{P}<0.05$; Fig. $1 \mathrm{C}-\mathrm{E})$ and osteoblastic differentiation (Fig. 1F and G), whereas inhibition of miR-186-5p exerted the opposite effects (Fig. 1C-G). Taken together, these results demonstrate that miR-186-5p participates in the disease course of OA through negatively regulating viability and differentiation of MSCs.

CXCL13 is a direct target of miR-186-5p in HMSCs. To determine the mechanism of how miR-186-5p suppressed differentiation of MSCs, the targets of miR-186-5p in HMSCs were determined. Bioinformatics analysis predicted that the 3'UTR of CXCL13 mRNA was a potential target of miR-186-5p (Fig. 2A and Table SII). Furthermore, RNA-seq was performed on MSCs from three patients with ON and three patients with OA patients. The potential functions of these differentially expressed mRNAs were identified using KEGG pathway analyses. The differentially abundant genes between $\mathrm{OA}$ and $\mathrm{ON}$ patients were significantly associated with Cytokine-cytokine receptor interaction pathways (Fig. S2). Specifically, expression of CXCL13 was downregulated in patients with ON (Fig. 2B; Table SIII). In addition, miR-186-5p expression was negatively correlated with CXCL13 mRNA levels in bone marrow samples from patients with ON patients (Fig. 2C). The dual-luciferase reporter assays suggested that transfection with miR-186-5p mimic or anti-miR-186-5p significantly reduced or increased, respectively the luciferase activity of WT CXCL13 3'UTR $(\mathrm{P}<0.05)$, whereas neither 
A

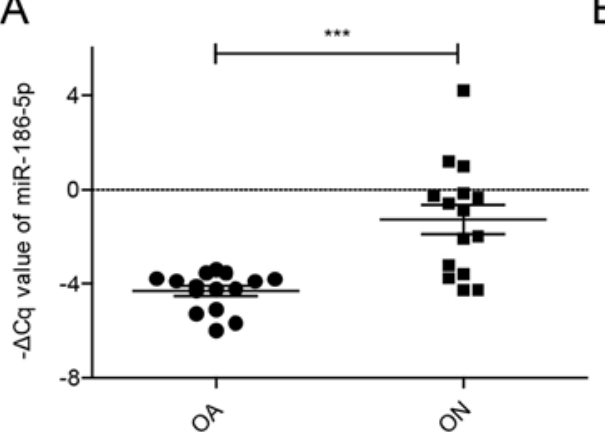

B

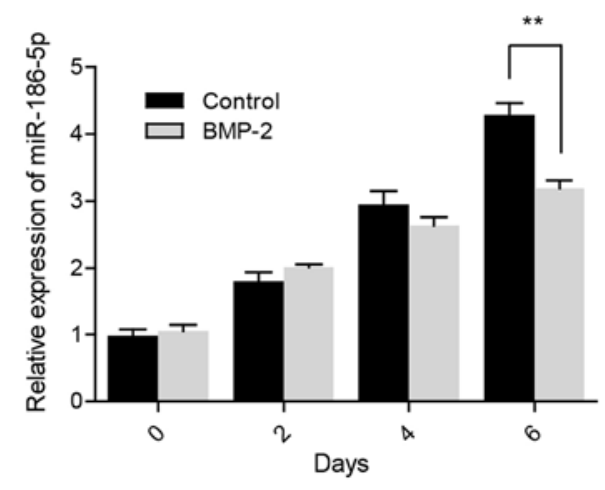

C

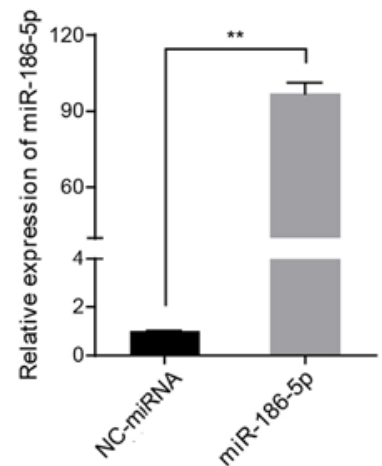

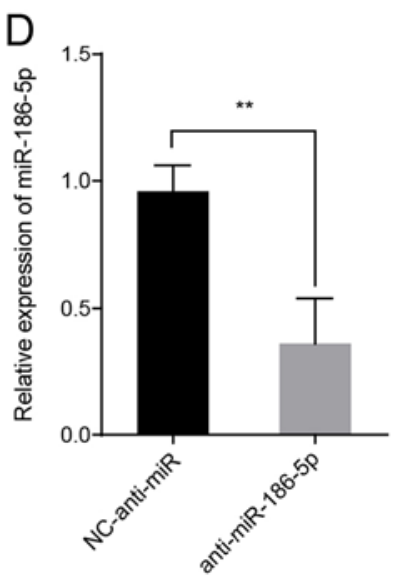

E

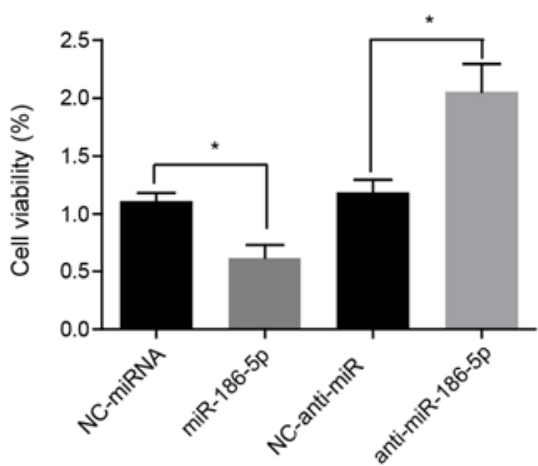

F
G

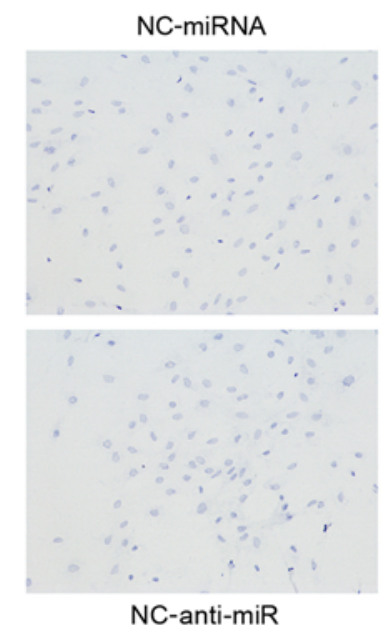

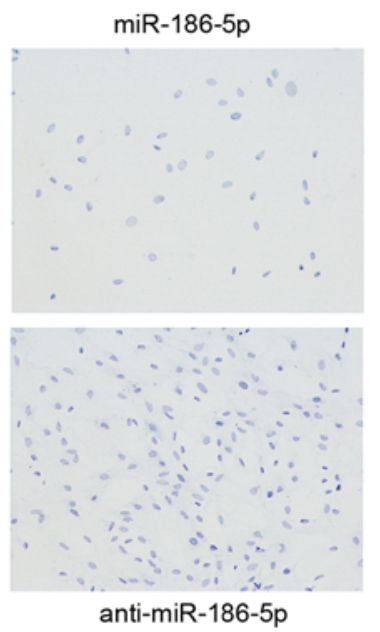
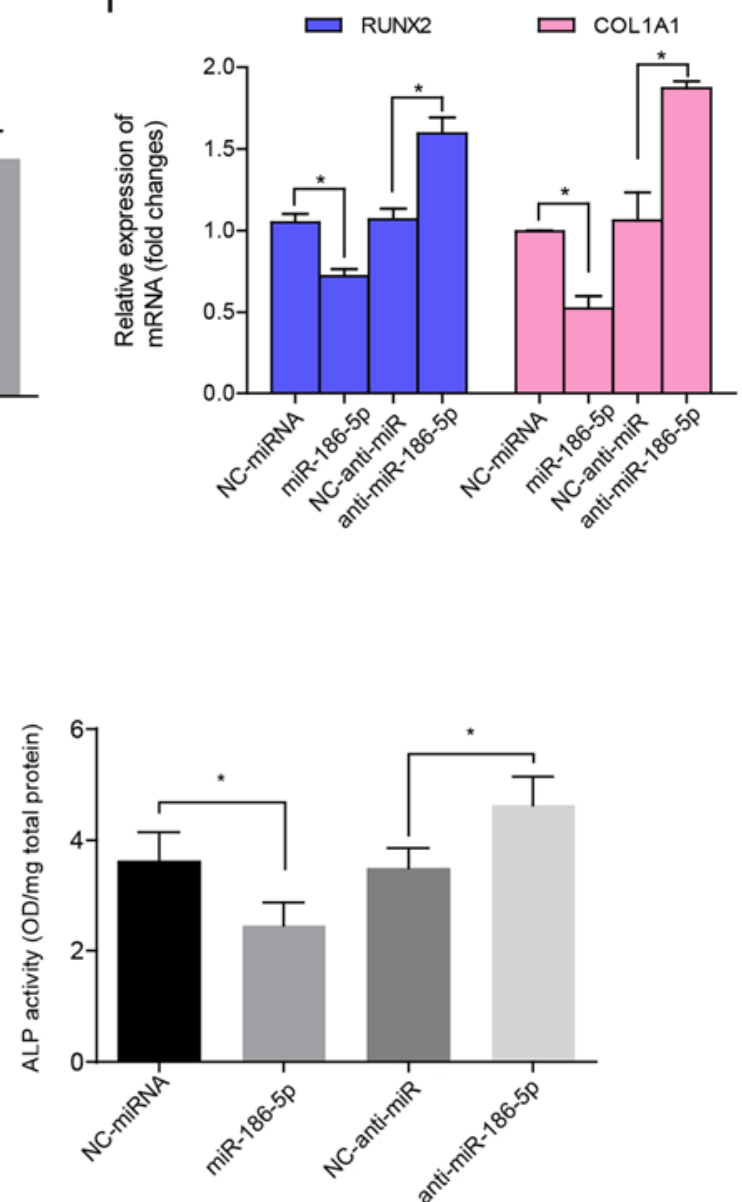

Figure 1. Expression of miR-186-5p is increased in patients with ONFH and modulates growth and differentiation in mesenchymal stem cells. (A) Expression of miR-186-5p was significantly increased in patients with ON compared with OA. ${ }^{* * *} \mathrm{P}<0.0001$. (B) Expression levels of miR-186-5p in HMSC-bm cells induced with BMP-2. Cells were harvested at the indicated time points and the expression levels of miR-186-5p was measured. There was a significant difference in the expression levels of miR-186-5p in cells induced with BMP-2 for 6 days. ${ }^{* *} \mathrm{P}<0.01$. Cells were transfected with either $(\mathrm{C}) \mathrm{miR}-186-5 \mathrm{p}$ mimics or (D) anti-miR-186-5p and treated with BMP-2 after $4 \mathrm{~h}$ for 6 days, after which the (C and D) levels of miR-186-5p or (E) viability of the cells was measured. ${ }^{*} \mathrm{P}<0.05$ and ${ }^{* *} \mathrm{P}<0.01$. (F) COL1A1 and RUNX2 expression levels were examined and (G) ALP activity was determined following transfection with either miR-186-5p mimics or anti-miR-186-5p. * $\mathrm{P}<0.05$. ONFH, osteonecrosis of femoral head; OA, osteoarthritis; BMP-2, bone morphogenetic protein-2; ALP, alkaline phosphatase; miR, microRNA; NC, negative control; OD, optical density; HMSC, human mesenchymal stem cells.

had any significant effects on the mut 3'UTR (Fig. 2D). These results suggest that miR-186-5p directly targets the 3'UTR of CXCL13. Transfection of miR-186-5p mimics or anti-miR-186-5p mimics into HMSCs cells or primary MSCs from patients with OA resulted in both mRNA and protein expression levels of CXCL13 being significantly reduced or increased, respectively ( $\mathrm{P}<0.05$; Fig. $2 \mathrm{E}-\mathrm{G})$. These results suggested that miR-186-5p directly targeted and abrogated CXCL13 expression in HMSCs.

miR-186-5p suppresses cell viability and osteoblastic differentiation by inhibiting CXCL13. Downregulation of CXCL13 mRNA levels was first observed in the RNA-seq data (Fig. 2B) and the results were validated in bone marrow tissues from 
A

Predicted consequential pairing of target region (top) and miRNA (bottom)

Position 733-740 of CXCL13 3' UTR

hsa-miR-186-5p

Position 733-740 of mutant CXCL13 3' UTR

B

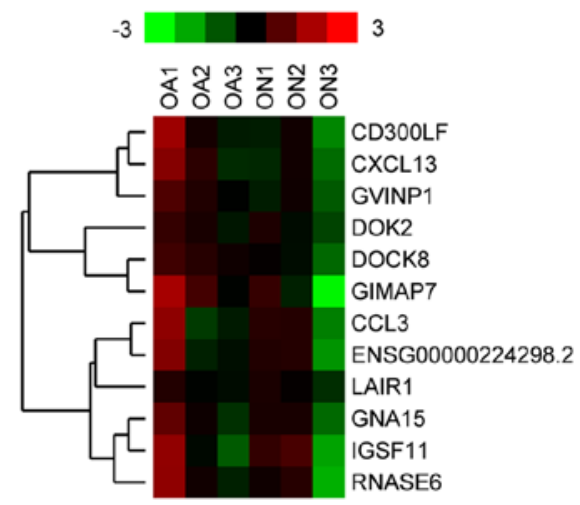

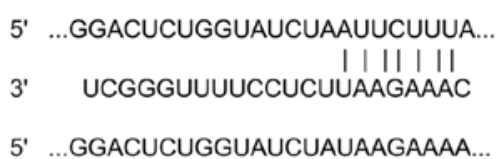

C

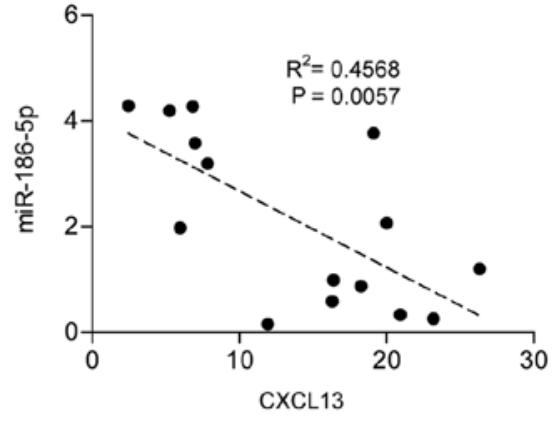

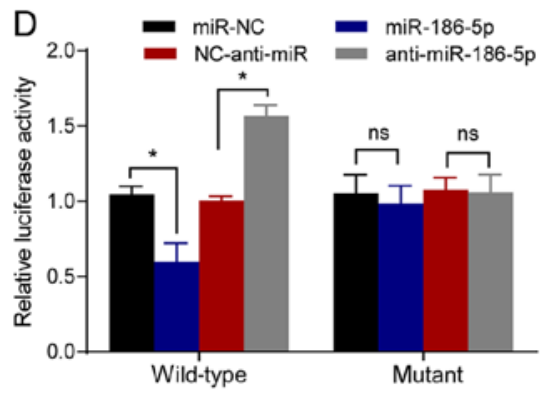

G

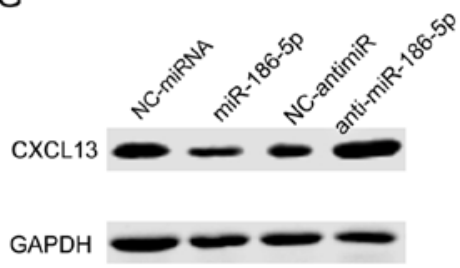

E

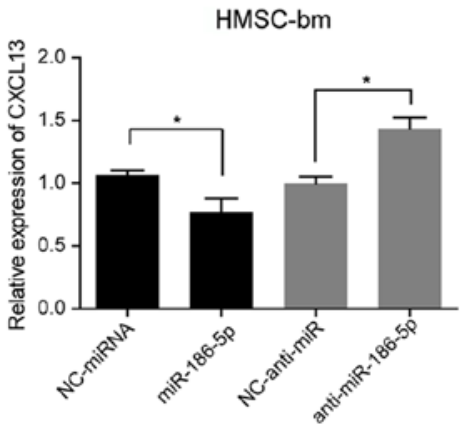

$\mathrm{H}$

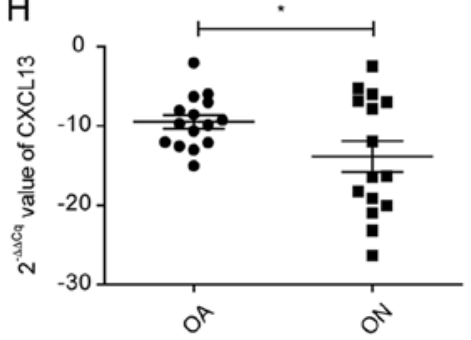

$\mathrm{F}$
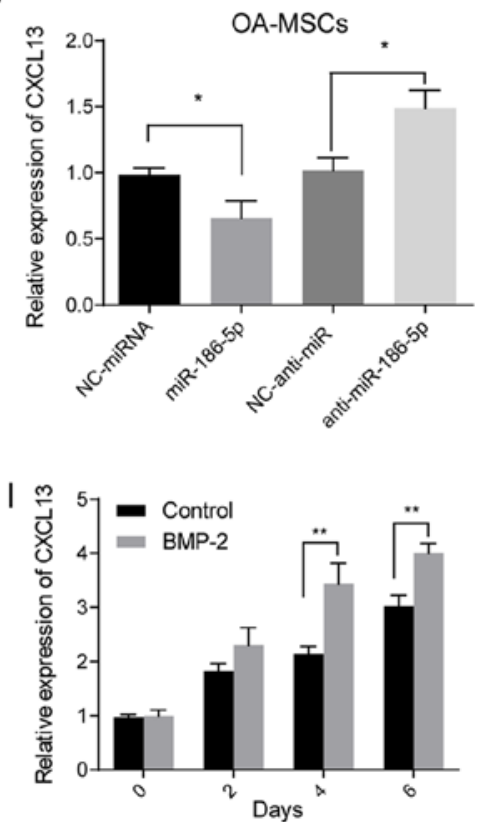

Figure 2. CXCL13 is a direct target of miR-186-5p in HMSCs. (A) Schematic diagram of the predicted miR-186-5p seed sequences in the 3'-UTRs of the wild type CXCL13 and mutant CXCL13 mRNAs. (B) A total of 12 genes were differentially expressed, out of 1,016 genes, at the mRNA level in clinical samples between patients with ON compared with OA. Gene expression data is presented as a matrix, in which columns represent individual mRNA samples and rows represent individual genes. Relative gene expression is presented as differing intensities of green and red according to the key. (C) Correlation analysis of miR-186-5p and CXCL13 expression in patients with ON. (D) Wild-type or mutant CXCL13 3'UTR was co-transfected with miR-186-5p mimics or anti-miR-186-5p in HMSC-bm cells and the relative activity of the luciferase reporter genes were measured after $24 \mathrm{~h}$. ${ }^{*} \mathrm{P}<0.05$. mRNA expression levels of CXCL13 in (E) HMSC-bm cells or (F) MSCs from OA patients (OA-MSCs, n=3) transfected with either miR-186-5p mimics or anti-miR-186-5p. * $<0.05$. (G) Protein expression levels of CXCL13 in HMSC-bm cells transfected with either miR-186-5p mimics or anti-miR-186-5p. (H) mRNA expression levels of CXCL13 in cultured primary cells obtained from patients with non-traumatic ON or OA. ${ }^{*} \mathrm{P}<0.05$. (I) Expression levels of CXCL13 in HMSC-bm cells induced with BMP-2. ${ }^{* *} \mathrm{P}<0.01$. UTR, untranslated region; ON, osteonecrosis; OA, osteoarthritis; BMP-2, bone morphogenetic protein-2; MSC, mesenchymal cells; $\mathrm{NC}$, negative control; HMSC, human mesenchymal stem cells; miR, microRNA; ns, non-significant.

patients with ON and OA patients using RT-qPCR (Fig. 2H). Compared with MSCs from the negative control group, CXCL13 expression levels increased faster when treated with BMP-2 for 6 days, suggesting that CXCL13 may serve an antagonistic role against miR-186-5p in MSC differentiation (Fig. 2I). Furthermore, the results showed that CXCL13 overexpression promoted cell viability and osteoblastic differentiation of MSCs, and reduction of CXCL13 mRNA expression levels had the opposite effects (Fig. 3A-E). To further investigate whether miR-186-5p modulated CXCL13 expression levels, rescue assays were performed. HMSCs cells were co-transfected with miR-186-5p mimic and a plasmid expressing CXCL13. As shown in Fig. 3, the inhibitory effects of miR-186-5p on CXCL13 were partially reversed after 
A

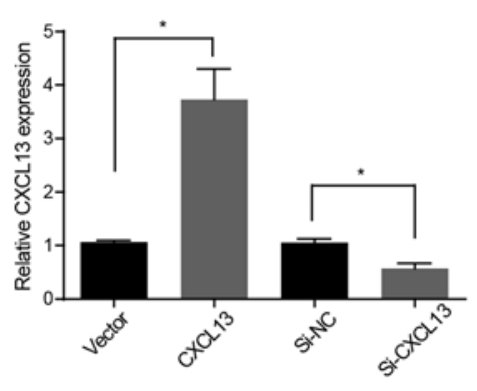

B

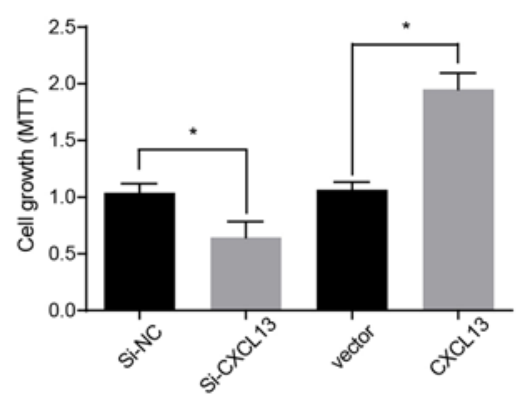

C

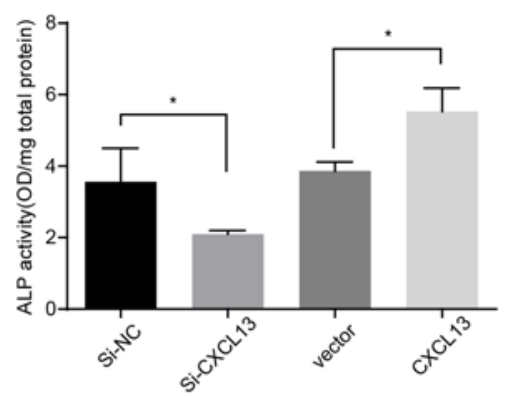

D

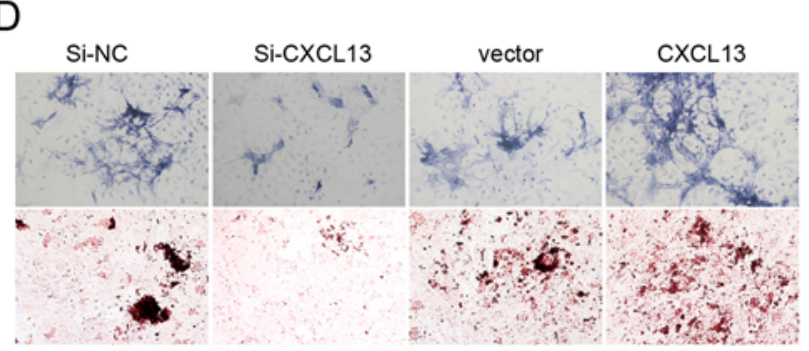

G

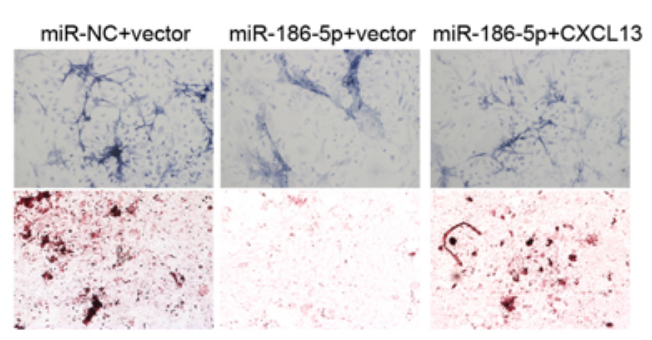

E

$\mathrm{H}$

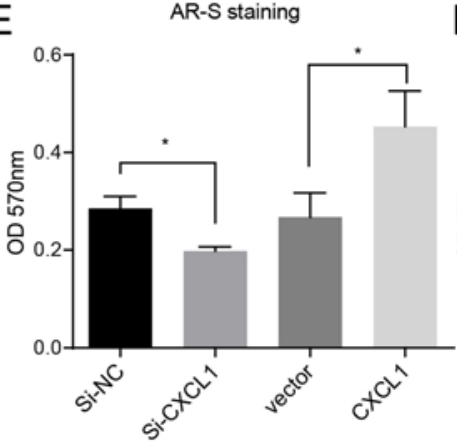

I
F AR-S staining

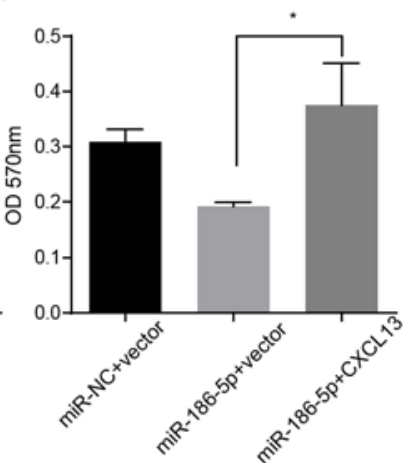

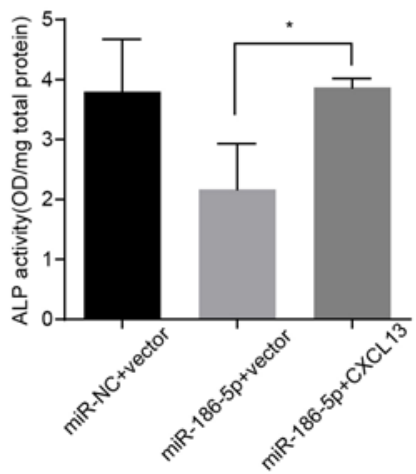

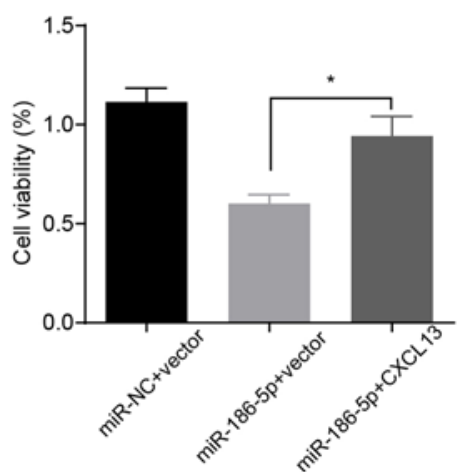

Figure 3. miR-186-5p decreases the viability of HMSC-bm cells and bone formation by negatively modulating CXCL13 expression. (A) Relative mRNA expression levels of CXCL13 $48 \mathrm{~h}$ after transfection with CXCL13, si-CXCL13 or negative control in HMSC-bm cells induced with BMP-2. A total of 6 days after treatment with BMP-2, the (B) cell viability, (C) ALP activity analysis and (D) cytology images and (E) ARS staining analysis were shown. Control and miR-186-5p mimics were co-transfected with or without pcDNA3.1-CXCL13 in HMSC-bm cells treated with BMP-2. After 6 days, (F) ARS staining and (G) cytology images, (H) ALP activity and (I) viability of cells were shown. Magnification, $\mathrm{x} 100$. $^{*} \mathrm{P}<0.05$. si, small interfering; NC, negative control; miR, microRNA; ALP, alkaline phosphatase; OD, optical density; ARS, Alizarin red S; BMP-2, bone morphogenetic protein-2; HMSC, human mesenchymal stem cells.

transfection with the plasmid (Fig. 3F-I). These results suggest that miR-186-5p targets and regulates CXCL13 in MSCs.

miR-186-5p/CXCL13 modulates AKT/ERK signaling to regulate cell growth and osteoblastic differentiation. It has been reported that CXCL13 promotes growth and invasion of colon cancer cells via the PI3K/AKT signaling pathway (31). To determine the underlying mechanism by which the miR-186-5p/CXCL13 axis in ON regulated cell growth and differentiation, the AKT signaling pathway in HMSC-bm cells was examined. Western blot analysis showed that overexpression of miR-186-5p significantly suppressed AKT/ERK signaling (Fig. 4). In addition, when the AKT signaling activator, $\mathrm{LiCl}$, was added to miR-186-5p-overexpressing
HMSC-bm cells, the MTT assays suggested that LiCl could restore the negative effects of miR-186-5p on HMSC-bm cell viability (Fig. 4A and B). Furthermore, transfection with the CXCL13 vector also partially rescued the growth of HMSC-bm cells when co-transfected with miR-186-5p (Fig. 4C and D). These data suggest that miR-186-5p/CXCL13 affects MSC growth through modulating PI3K/AKT signaling.

\section{Discussion}

Accumulating evidence has shown that miRNAs function by modulating various biological processes in human MSCs. In the etiopathogenesis of non-traumatic ON, certain microRNAs, such as miR-17-5p, miR-125 and miR-135 have been identified as 

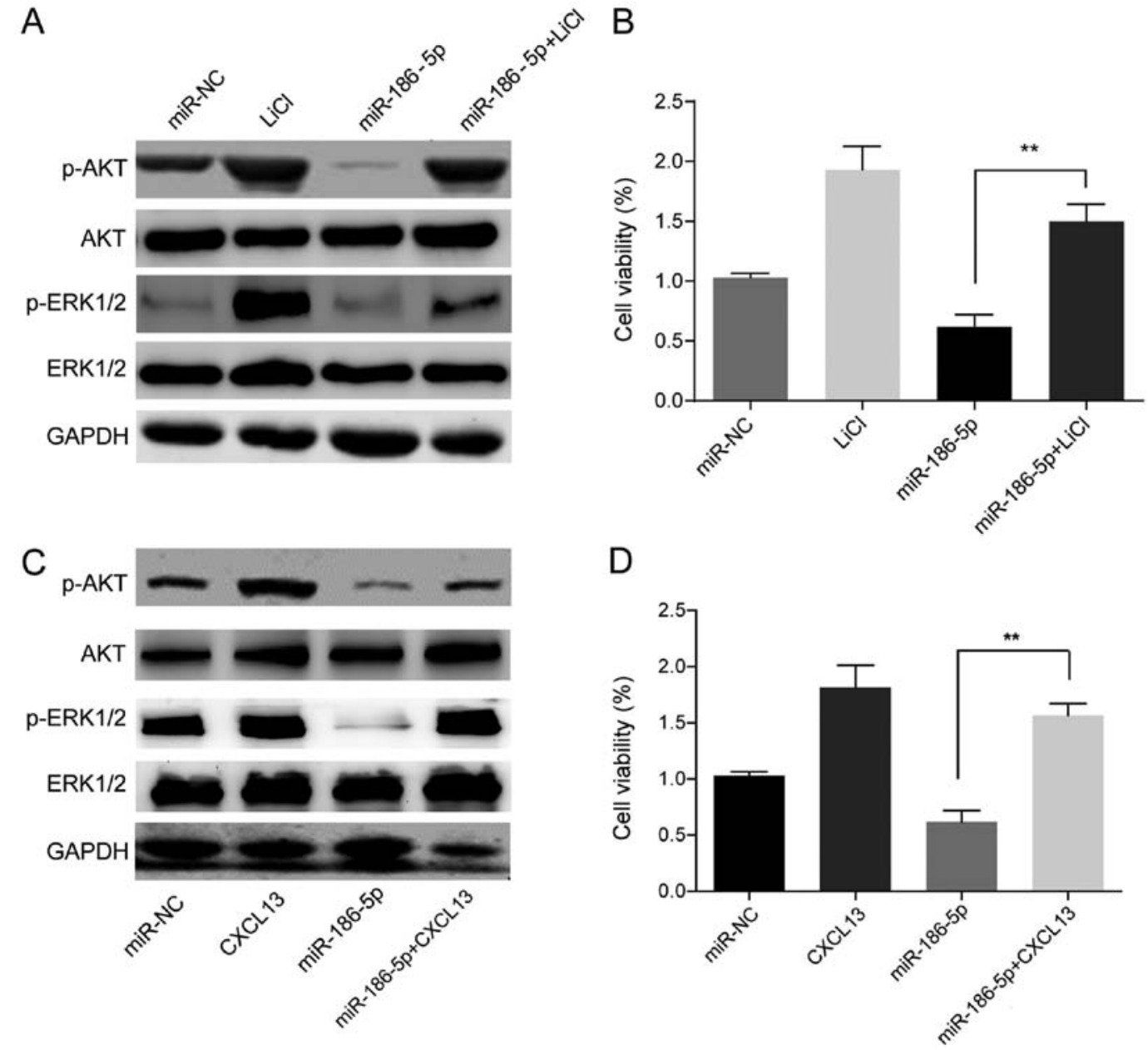

Figure 4. miR-186-5p inhibits CXCL13-mediated downstream PI3K/AKT signaling. (A) HMSC-bm cells were transfected with miR-186-5p mimics and treated with $\mathrm{LiCl}$ at day 1 in vitro. Total and p-AKT, ERK1/2 protein expression levels were assessed using western blotting at day 4. (B) Cell viability was determined using an MTT assay after 6 days of treatment. (C) HMSC-bm cells were transfected with control or miR-186-5p mimics and CXCL13 at day 1. Total and p-AKT, ERK1/2 levels were assessed using western blotting at day 4. (D) Cell viability was determined using MTT assays at day 6. ${ }^{* *} \mathrm{P}<0.01$. miR, microRNA; NC, negative control; HMSC, human mesenchymal stem cells; p-AKT, phosphorylated-protein kinase B; extracellular signal regulated kinase.

modulators of cell growth and differentiation $(32,33)$, highlighting the importance of miRNAs in osteonecrosis diagnosis and treatment. However, the functional significance of miRNAs is not yet completely understood. In the present study, it was demonstrated that expression of miR-186-5p was increased in the HMSCs of patients with $\mathrm{ON}$ and served important roles in regulating cell growth and osteoblastic differentiation through directly targeting CXCL13. The data of the present study demonstrated that the expression of miR-186-5p was altered when CXCL13 expression was increased during osteoblast differentiation of HMSCs, suggesting their involvement in bone development. Furthermore, miR-186-5p overexpression suppressed activation of the AKT/ERK signaling pathway. Specific activation of AKT/ERK signaling rescued the effects of miR-186-5p in HMSCs.

Chemokines are the largest family of cytokines, with at least 47 members which are divided into four major categories, the $\mathrm{CX} 3 \mathrm{C}, \mathrm{CXC}, \mathrm{CC}$ and $\mathrm{C}$ subfamilies, based on the spacing and number of conserved cysteine residues in the amino acid sequences. Chemokines bind to seven transmembrane-spanning G-protein-coupled receptors to exert influence on various biological functions (34). CXCL13 directly modulates cellular proliferation and viability induces migration of MSCs to move to the injured area and induces differentiation of osteoblasts (35-37).
Although CXCL13 was considered to be a critical regulator of MSC differentiation, none of characteristics were significantly correlated with CXCL13 expression in the 15 patients enrolled in the present study (Data not shown), given the small sample size. This result should be further explored within a larger cohort.

The RNA-seq analysis results showed that CXCL13 expression was downregulated in HMSCs of patients with ON. In addition, CXCL13 mRNA expression levels were negatively correlated with miR-186-5p expression levels in bone marrow samples in patients with ON. miR-186-5p downregulated mRNA as well as the protein expression levels of CXCL13 in HMSCs and increasing the levels of CXCL13 partially restored miR-186-5p-hindered osteoblastic differentiation, and cell viability. The present study also predicted the underlying functions of differentially abundant mRNAs using KEGG pathway analyses. These findings corroborated the hypothesis that one of the most significantly important pathways in the development of osteonecrosis was cytokine-cytokine receptor interaction, which was also identified by previous studies $(38,39)$. Taken together, the present study highlighted the important role of miRNA-CXCL13 regulation in patients with non-traumatic $\mathrm{ON}$ and may serve as potential therapeutic targets for treating patients with $\mathrm{ON}$. 


\section{Acknowledgements}

The authors thank Professor HL Jin from Cancer Centre, Wuhan Union Hospital for helpful and valuable discussions, Dr Xiao-Bo Feng and Dr Wei Tong from Department of Orthopedics, Wuhan Union Hospital for the technical assistance.

\section{Funding}

The present study was supported by The National Natural Science Foundation of China (grant nos. 81702193, 81572161, 81602107 and 81672155).

\section{Availability of data and materials}

The datasets used and/or analyzed during the current study are available from the corresponding author on reasonable request.

\section{Authors' contributions}

WHX and JL obtained human specimens, provided support with experimental techniques and wrote the manuscript. HTT prepared all samples and clinical data. RYW performed bioinformatics analysis of experimental data. YF performed bioinformatics analysis of clinical data. JT and JJ designed research, performed all experiments, conceived the study and supervised all experiments.

\section{Ethics approval and consent to participate}

The experimental protocol was established, according to the ethical guidelines of the Helsinki Declaration and was approved by the Human Ethics Committee of Wuhan Union Hospital (approval no. 17S001).

\section{Patient consent for publication}

Written informed consent was obtained from all individual participants.

\section{Competing interests}

The authors declare that they have no competing interests.

\section{References}

1. Liu Y, Wu J, Zhu Y and Han J: Therapeutic application of mesenchymal stem cells in bone and joint diseases. Clin Exp Med 14: 13-24, 2014.

2. Mont MA, Marulanda GA, Jones LC, Saleh KJ, Gordon N, Hungerford DS and Steinberg ME: Systematic analysis of classification systems for osteonecrosis of the femoral head. J Bone Joint Surg Am 88 (Suppl 3): S16-S26, 2006.

3. Zhao DW, Yu M, Hu K, Wang W, Yang L, Wang BJ, Gao XH, Guo YM, Xu YQ, Wei YS, et al: Prevalence of Nontraumatic osteonecrosis of the femoral head and its associated risk factors in the Chinese population: Results from a nationally representative survey. Chin Med J (Engl) 128: 2843-2850, 2015.

4. Yamamoto T, DiCarlo EF and Bullough PG: The prevalence and clinicopathological appearance of extension of osteonecrosis in the femoral head. J Bone Joint Surg Br 81: 328-332, 1999.

5. Tan G, Kang PD and Pei FX. Glucocorticoids affect the metabolism of bone marrow stromal cells and lead to osteonecrosis of the femoral head: A review. Chin Med J (Engl) 125: 134-139, 2012 .
6. Seamon J, Keller T, Saleh J and Cui Q: The pathogenesis of nontraumatic osteonecrosis. Arthritis 2012: 601763, 2012.

7. Feng Y, Yang SH, Xiao BJ, Xu WH, Ye SN, Xia T, Zheng D, Liu XZ and Liao YF: Decreased in the number and function of circulation endothelial progenitor cells in patients with avascular necrosis of the femoral head. Bone 46: 32-40, 2010.

8. Li Z, Liao W, Zhao Q, Liu M, Xia W, Yang Y and Shao N: Angiogenesis and bone regeneration by allogeneic mesenchymal stem cell intravenous transplantation in rabbit model of avascular necrotic femoral head. J Surg Res 183: 193-203, 2013.

9. Zhao D, Cui D, Wang B, Tian F, Guo L, Yang L, Liu B and Yu X: Treatment of early stage osteonecrosis of the femoral head with autologous implantation of bone marrow-derived and cultured mesenchymal stem cells. Bone 50: 325-330, 2012.

10. Gangji V, De Maertelaer V and Hauzeur JP: Autologous bone marrow cell implantation in the treatment of non-traumatic osteonecrosis of the femoral head: Five year follow-up of a prospective controlled study. Bone 49: 1005-1009, 2011.

11. Yan Z, Hang D, Guo $C$ and Chen Z: Fate of mesenchymal stem cells transplanted to osteonecrosis of femoral head. J Orthop Res 27: 442-446, 2009.

12. Jiang S, Zu Y, Fu Y, Zhang Y and Efferth T: Activation of the mitochondria-driven pathway of apoptosis in human PC-3 prostate cancer cells by a novel hydrophilic paclitaxel derivative, 7-xylosyl-10-deacetylpaclitaxel. Int J Oncol 33: 103-111, 2008.

13. Nishimori S, Tanaka Y, Chiba T, Fujii M, Imamura T, Miyazono K, Ogasawara T, Kawaguchi H, Igarashi T, Fujita T, et al: Smad-mediated transcription is required for transforming growth factor-beta 1-induced p57(Kip2) proteolysis in osteoblastic cells. J Biol Chem 276: 10700-10705, 2001.

14. Fabian MR, Sonenberg N and Filipowicz W: Regulation of mRNA translation and stability by microRNAs. Annu Rev Biochem 79: 351-379, 2010.

15. Guo H, Ingolia NT, Weissman JS and Bartel DP: Mammalian microRNAs predominantly act to decrease target mRNA levels. Nature 466: 835-840, 2010.

16. Lau NC, Lim LP, Weinstein EG and Bartel DP: An abundant class of tiny RNAs with probable regulatory roles in Caenorhabditis elegans. Science 294: 858-862, 2001.

17. Lagos-Quintana M, Rauhut R, Lendeckel W and Tuschl T: Identification of novel genes coding for small expressed RNAs. Science 294: 853-858, 2001.

18. Gustafson D, Tyryshkin K and Renwick N: microRNA-guided diagnostics in clinical samples. Best Pract Res Clin Endocrinol Metab 30: 563-575, 2016.

19. Yamasaki K, Nakasa T, Miyaki S, Yamasaki T, Yasunaga Y and Ochi M: Angiogenic microRNA-210 is present in cells surrounding osteonecrosis. J Orthop Res 30: 1263-1270, 2012.

20. van Wijnen AJ, van de Peppel J, van Leeuwen JP, Lian JB, Stein GS, Westendorf JJ, Oursler MJ, Im HJ, Taipaleenmäki H, Hesse E, et al: MicroRNA functions in osteogenesis and dysfunctions in osteoporosis. Curr Osteoporos Rep 11: 72-82, 2013.

21. Cai J, Wu J, Zhang H, Fang L, Huang Y, Yang Y, Zhu X, Li R and Li M: miR-186 downregulation correlates with poor survival in lung adenocarcinoma, where it interferes with cell-cycle regulation. Cancer Res 73: 756-766, 2013.

22. Li H, Yin C, Zhang B, Sun Y, Shi L, Liu N, Liang S, Lu S, Liu Y, Zhang J, et al: PTTG1 promotes migration and invasion of human non-small cell lung cancer cells and is modulated by miR-186. Carcinogenesis 34: 2145-2155, 2013.

23. Kim SY, Lee YH and Bae YS: MiR-186, miR-216b, miR-337-3p, and miR-760 cooperatively induce cellular senescence by targeting $\alpha$ subunit of protein kinase CKII in human colorectal cancer cells. Biochem Biophys Res Commun 429: 173-179, 2012.

24. Pike KA, Hutchins AP, Vinette V, Théberge JF, Sabbagh L, Tremblay ML and Miranda-Saavedra D: Protein tyrosine phosphatase $1 \mathrm{~B}$ is a regulator of the interleukin-10-induced transcriptional program in macrophages. Sci Signal 7: ra43, 2014.

25. Trapnell C, Williams BA, Pertea G, Mortazavi A, Kwan G, van Baren MJ, Salzberg SL, Wold BJ and Pachter L: Transcript assembly and quantification by RNA-Seq reveals unannotated transcripts and isoform switching during cell differentiation. Nat Biotechnol 28: 511-515, 2010.

26. Anders S and Huber W: Differential expression analysis for sequence count data. Genome Biol 11: R106, 2010. 
27. Hutchins AP, Jauch R, Dyla M and Miranda-Saavedra D: Glbase: A framework for combining, analyzing and displaying heterogeneous genomic and high-throughput sequencing data. Cell Regen (Lond) 3: 1, 2014.

28. Livak KJ and Schmittgen TD: Analysis of relative gene expression using real-time quantitative PCR and the 2(-Delta Delta C(T)) method. Methods 25: 402-408, 2001.

29. Hu L, Chen L, Li L, Sun H, Yang G, Chang Y, Tu Q, Wu M and Wang H: Hepatitis B virus X protein enhances cisplatin-induced hepatotoxicity via a mechanism involving degradation of Mcl-1. J Virol 85: 3214-3228, 2011.

30. Lu K, Zeng D, Zhang Y, Xia L, Xu L, Kaplan DL, Jiang X and Zhang F: BMP-2 gene modified canine bMSCs promote ectopic bone formation mediated by a nonviral PEI derivative. Ann Biomed Eng 39: 1829-1839, 2011.

31. Zhu Z,Zhang X, Guo H,Fu L,Pan G and Sun Y: CXCL13-CXCR5 axis promotes the growth and invasion of colon cancer cells via PI3K/AKT pathway. Mol Cell Biochem 400: 287-295, 2015.

32. Jia J, Feng X, Xu W, Yang S, Zhang Q, Liu X, Feng Y and Dai Z: MiR-17-5p modulates osteoblastic differentiation and cell proliferation by targeting SMAD7 in non-traumatic osteonecrosis. Exp Mol Med 46: e107, 2014.

33. Jing D, Hao J, Shen Y, Tang G, Li ML, Huang SH and Zhao ZH: The role of microRNAs in bone remodeling. Int J Oral Sci 7 : 131-143, 2015.

34. Rüegg C,Hasmim M,Lejeune FJ and Alghisi GC: Antiangiogenic peptides and proteins: From experimental tools to clinical drugs. Biochim Biophys Acta 1765: 155-177, 2006.
35. Lisignoli G, Toneguzzi S, Piacentini A, Cristino S, Grassi F Cavallo $\mathrm{C}$ and Facchini A: CXCL12 (SDF-1) and CXCL13 (BCA-1) chemokines significantly induce proliferation and collagen type I expression in osteoblasts from osteoarthritis patients. J Cell Physiol 206: 78-85, 2006.

36. Cristino S, Piacentini A, Manferdini C, Codeluppi K, Grassi F, Facchini A and Lisignoli G: Expression of CXC chemokines and their receptors is modulated during chondrogenic differentiation of human mesenchymal stem cells grown in three-dimensional scaffold: Evidence in native cartilage. Tissue Eng Part A 14: 97-105, 2008.

37. Tian F, Ji XL, Xiao WA, Wang B and Wang F: CXCL13 promotes osteogenic differentiation of mesenchymal stem cells by inhibiting miR-23a expression. Stem Cells Int 2015: 632305, 2015.

38. Adapala NS and Kim HW: Comprehensive genome-wide transcriptomic analysis of immature articular cartilage following ischemic osteonecrosis of the femoral head in piglets. PLoS One 11: e0153174, 2016

39. Yuan C and Cai J: Time-series expression profile analysis of fracture healing in young and old mice. Mol Med Rep 16: 4529-4536.

This work is licensed under a Creative Commons Attribution-NonCommercial-NoDerivatives 4.0 International (CC BY-NC-ND 4.0) License. 